\title{
Trabajo en la infancia. Una aproximación al trabajo doméstico y extradoméstico en México desde el Módulo de Trabajo Infantil 2015*
}

\section{Work in childhood.}

An approach to domestic and extra-domestic work

in Mexico from the Child

Labor Module 2015

Carmen Monzerrat Valdez Navarro**

Recibido: 13 de marzo de 2019

Revisado: 3 de abril de 2019

Aprobado: 10 de junio de 2019

* Cómo citar este artículo: Valdez, C. M. (2019). Trabajo en la infancia. Una aproximación al trabajo doméstico y extradoméstico en México desde el Módulo de Trabajo Infantil 2015. Revista CIFE: Lecturas de Economía Social, 21(34), 47-68

** Maestra en demografía por El Colegio de México. Correo electrónico: cmvaldez@colmex.mx. Orcid: https://orcid.org/0000-0001-5486-4988 


\section{Resumen}

El trabajo infantil se entiende como aquel que ocurre en el ámbito extradoméstico; sin embargo, levantamientos de información y conceptualizaciones actuales permiten realizar aproximaciones a las labores que los niños, niñas y adolescentes realizan dentro de sus hogares y pueden vulnerar su desarrollo. Esta investigación tiene por objetivo estimar el efecto de las características de los hogares sobre la probabilidad que tienen niños y adolescentes mexicanos de integrarse - o no- en el trabajo doméstico o extradoméstico. Se emplea como fuente de datos el Módulo de Trabajo Infantil (MTI) 2015, anexo a la Encuesta Nacional de Ocupación y Empleo. Los resultados muestran que existen diferencias significativas entre el tipo de hogar y la probabilidad de ingreso al trabajo doméstico o extradoméstico, dependiendo del sexo del jefe(a) de la unidad ${ }^{1}$.

Palabras clave: niños, jóvenes, estructura familiar.

Clasificación JEL: J13, J12.

\section{Abstract}

Child labor is commonly understood as that which occurs in the extra-domestic environment; however, information surveys and current conceptualizations allow us to make approximations to the tasks that children and adolescents perform within their homes and may violate their development. The purpose of this research is to estimate the effect of variables at the meso-level on the probability that Mexican children and adolescents have of integrating-or not-into domestic or extra-domestic work, with special emphasis on the type of household arrangement in which they live and the sex of the head of the household. The Child Labor Module (MTI) 2015, annexed to the Survey of Occupation and Employment, is used as a data source. The results show significant differences between the type of household and the probability of entering domestic or extra-domestic work depending on the sex of the head of the unit.

Keywords: Children, youth, family structure.

Classification JEL: J13, J12. 


\section{Introducción}

Niños, niñas y adolescentes (NNA) han participado de manera histórica en actividades domésticas y extradomésticas que pueden vulnerar su desarrollo. Gradualmente, alrededor del mundo, se han implementado restricciones que buscan regular de manera legal la realización de dichas actividades; sin embargo, existen distintos retos para la medición y conceptualización del trabajo infantil ${ }^{2}$.

Según los resultados del Módulo de Trabajo Infantil 2015 (MTI, 2015) se estima que en el país habitaban alrededor de 29370227 NNA; casi dos millones se desempeñaban en actividades económicas, ya sea bajo la edad mínima de acceso al empleo o en ocupaciones peligrosas ${ }^{3}$. Así mismo, en el MTI 2015 se reportó que poco más de millón y medio realizaban quehaceres domésticos durante horarios prolongados o en condiciones peligrosas ${ }^{4}$.

Actualmente, se considera que las configuraciones "tradicionales" de los hogares, conformadas por pareja e hijos, han perdido importancia relativa respecto a otro tipo de convivencias, la proporción de hogares con jefatura femenina se ha incrementado de manera importante, pues diversos factores del orden demográfico, social y económico han propiciado su conformación: la formación de uniones consensuales y su disolución, tendencias en la migración interna e internacional de los varones, violencia doméstica, incremento de la escolaridad de las mujeres; así como la inmersión de las mujeres en mercados laborales, entre otros (García y Oliveira, 2017).

Este estudio tiene como objetivo estimar el efecto del arreglo de hogar en que habitan NNA sobre la probabilidad de realizar trabajo dentro de la esfera doméstica o extradoméstica, e identificar si estos efectos se diferencian de acuerdo con el sexo del jefe(a) del hogar. Se argumenta que las configuraciones de los hogares pueden propiciar o resguardar a NNA de realizar trabajo doméstico o extradoméstico, ya que se considera que los

2 Este artículo se conforma como un extracto de la tesis para obtener el grado de maestra en Demografía por parte de El Colegio de México: "Trabajo en la infancia en los ámbitos doméstico y extradoméstico: una aproximación desde las características de los hogares. México 2015” (Valdez, 2018).

3 En el caso mexicano la edad mínima de acceso al empleo se incrementó en el año 2014 de los 14 a los 15 años (Diario Oficial de la Federación [DOF], 2014). Las ocupaciones peligrosas contemplan que NNA realicen actividades en lugares peligrosos e insalubres, que las actividades reportadas los expongan a riesgos sobre su salud o moral, que no cuenten con permisos y certificados de salud y que realicen actividades en horarios nocturnos o prolongados; las delimitaciones varían según la legislación de cada entidad federativa.

4 En el documento metodológico del MTI 2015 se establece que no existe un criterio estandarizado para determinar el número de horas que pueden afectar el desarrollo de NNA; sin embargo, se consideran los siguientes criterios para su clasificación: a) 5 a 11 años, superior a 14 horas, b) de 12 a 14 años, mayor de 28 horas, c) 15 años, más de 36 horas y d) 16 y 17 años mayor a 48 horas.

Respecto a quehaceres domésticos en condiciones peligrosas, esta institución retoma elementos como cargar cosas pesadas, presentar dolores de espalda o musculares, cansancio o agotamiento, problemas respiratorios o intoxicación y no cumplir con actividades escolares; estos se preguntan a los informantes durante el levantamiento del cuestionario. 
hogares implementan estrategias para lograr su sobrevivencia, las cuales pueden incluir la participación de infantes y adolescentes en actividades de riesgo.

El artículo se estructura de la siguiente manera: primero, se aborda la conceptualización del trabajo infantil en los ámbitos doméstico y extradoméstico; después, se profundiza en las características de los hogares como factores que pueden propiciar o desincentivar el trabajo en la infancia; enseguida, se describe la fuente de información, así como el método empleado para dar respuesta a la pregunta de investigación planteada (modelos logísticos multinomiales). Finalmente, se presentan los resultados que arrojan los modelos generados, así como las conclusiones y discusión de la investigación.

\section{La conceptualización del trabajo en la infancia}

Históricamente se ha registrado la participación infantil y adolescente en actividades domésticas y extradomésticas actualmente consideradas de riesgo para la salud y desarrollo físico y mental. Edgell (201 1), por ejemplo, aborda cómo los cambios en el paradigma económico adoptado en distintos momentos del tiempo, han reconfigurado la participación de los miembros del hogar y su relación con los medios requeridos para lograr su supervivencia. Dicha participación ha cambiado dependiendo del contexto histórico.

La regulación de la participación laboral de niños, niñas y adolescentes es relativamente reciente; la Organización Internacional del Trabajo (OIT) es uno de los organismos principales que ha establecido acuerdos y recomendaciones para eliminar en cada uno de los países, de manera paulatina, lo que se define como trabajo infantil (TI) (Pérez, 2015). Sin embargo, dicha institución no se ha encontrado exenta de dificultades en la conceptualización, pues en principio la definición del TI se encontraba relacionada con la actividad económica:

[El T] comprende la mayoría de las actividades productivas realizadas por niños, destinadas o no al mercado, remuneradas o no, por pocas horas o a tiempo completo, de manera ocasional o regular, legal o ilegal; excluye las tareas que los niños realizan en su propio hogar y las actividades escolares. (OIT, 2006, p. 6)

El concepto de trabajo infantil se construyó a partir del convenio número 138 sobre la edad mínima de ingreso al empleo (OIT, 2006). Este entró en vigor en junio del año 1976 y en él se establecía el compromiso de los países miembros a elevar de manera gradual la edad mínima en la que se podía ingresar al mercado laboral con el fin de promover "el más completo desarrollo físico y mental de los menores" (artículo 1).

El reconocimiento del trabajo no remunerado desde una perspectiva teórico-conceptual tuvo lugar en los años setenta, en esta época, movimientos feministas discuten acerca de los aportes del trabajo doméstico sobre la reproducción social y la fuerza de trabajo, 
después de esta época se ha profundizado en las discusiones de su conceptualización (García, 2019). No obstante, las actividades domésticas se encontraban excluidas de la definición de TI hasta la XVIII Conferencia Internacional de Estadísticas del Trabajo (XVIII CIET) llevada a cabo en el año 2008, donde se presentó una resolución con el fin de establecer normas para recopilar, compilar y analizar estadísticas generadas en los países para su medición. En esta conferencia se llegó a un consenso sobre aquellas formas de participación permitidas y no permitidas entre la población de 5 a 17 años, y se enmarcaron diferencias conceptuales existentes entre aquellos NNA considerados como ocupados en la producción económica y los que realizan otras actividades productivas (OIT, 2008). Es a partir de este momento donde, al menos en términos de recopilación estadística, se integran también las actividades realizadas dentro de la esfera doméstica.

Con fines de medición estadística los niños, niñas y adolescentes ocupados se clasifican en ocupados dentro de la producción económica y en ocupados dentro de otras actividades productivas. Es decir, los primeros se encuentran dentro de la Frontera General de Producción (FGP) del Sistema de Cuentas Nacionales (SCN), mientras que los segundos se localizan fuera de dicha frontera ${ }^{5}$.

Dentro de la FGP se considera que NNA se encuentran en situación de TI si realizan alguna de las peores formas de trabajo infantil (formas incuestionablemente peores de trabajo infantil y trabajo peligros) ${ }^{6}$, así como quienes realizan trabajo bajo la edad mínima ${ }^{7}$. Fuera de la FGP, y en situación vulnerable, se encuentran los NNA que realizan servicios domésticos no remunerados peligrosos ${ }^{8}$. En adelante, se le llamará trabajo en la infancia a ambas formas de trabajo definidas en este párrafo.

\section{Las características del hogar asociadas al trabajo en la infancia}

De acuerdo con la disposición de recursos (humanos y materiales), desde el enfoque de estrategias familiares de vida, en el interior de los hogares se toman decisiones mediante las cuales se busca sostener dichas unidades (Contreras, 2017), la organización económica, social, así como su estructura y composición son determinantes en la implementación de estrategias que definen la participación de sus miembros en diversas actividades.

5 Dentro de la FGP se encuentran los NNA que: 1) realizan trabajo ligero permitido, 2) realizan trabajo no señalado como peores formas de trabajo infantil y los que realizan trabajo infantil.

6 Las formas incuestionablemente peores de trabajo infantil comprenden actividades ilícitas, prostitución, esclavitud, pornografia, etc. (OIT, 2008).

7 La edad mínima de ingreso al empleo se establece en la ratificación del convenio 138 de la OIT, en esta se especifica que "la edad mínima de admisión al empleo no deberá ser inferior a la edad en que cesa la obligación escolar, o en todo caso, a 15 años".

8 Fuera de la FGP se encuentran los NNA que: 1) realizan servicios domésticos no remunerados peligrosos y 2) los que realizan otras actividades fuera de la frontera general de producción. 
Se han documentado distintas dimensiones que afectan la estructura, configuración y dinámica interna de los hogares. En ese sentido, Ariza y Oliveira (2001) identifican que las transformaciones socioeconómicas, culturales y demográficas han llevado a que las sociedades latinoamericanas experimenten cambios importantes en los hogares nucleares tradicionales (hogar nuclear biparental con jefatura masculina y proveedor único), ya que este tipo de hogares, a pesar de ser predominantes, han perdido poco a poco importancia relativa respecto a otros tipos de hogar. Por otro lado, la conformación de familias extensas y compuestas ha sido persistente en la región debido a factores económicos o culturales que las propician (Ariza y Oliveira, 2007).

Respecto a la composición del hogar por el sexo del jefe(a), en las últimas décadas los hogares con jefatura femenina se han incrementado de manera importante. Estos pasaron de representar $15.3 \%$ en 1970 a $29 \%$ durante 2015 (García y Oliveira, 2014; Encuesta Intercensal [INEGI 2015). La manera en que se estudia la jefatura femenina en los hogares ha transitado del mero análisis sociodemográfico de las unidades domésticas a estudios que se enfocan en las características de este tipo de jefaturas, dada la preocupación existente sobre conformaciones familiares distintas a la "familia tradicional" y al bienestar de sus miembros (Acosta, 2001).

Se ha documentado la influencia de distintos factores a nivel macro (características contextuales), meso (características del hogar) y micro (características individuales) que tienen efectos sobre la probabilidad de que NNA participen en labores domésticas y extradomésticas; se considera que, bajo ciertas condiciones, estas actividades podrían traducirse en daños para quienes las realizan en diferentes dimensiones, como su salud y desarrollo (Briceño y Pinzón, 2004). Este documento busca resaltar la importancia de la configuración y el sexo del jefe(a) del hogar y la manera en que esta construcción impacta en la probabilidad de realizar - o no- trabajo en la infancia.

El impacto del tipo de hogar sobre las actividades que realizan niños, niñas y adolescentes ha sido discutido por diferentes autores. Mier y Rabell (2004), por ejemplo, al relacionar el tipo de unidad familiar con el sector socioeconómico de pertenencia señalan que "las estructuras familiares de cada uno de los sectores pueden estar asociadas a diferentes patrones de relaciones intrafamiliares y, por ende, tener un efecto distinto en las actividades de los jóvenes" (Mier et al., 2004, p.168). De igual manera, Estrada (2005) encuentra que la probabilidad de que NNA trabajen se encuentra influenciada por características relacionadas con las familias, y que esta es diferencial de acuerdo con el tipo de trabajo que se realice (doméstico o extradoméstico).

Respecto a la jefatura de los hogares, resulta importante implementar en los análisis el estudio de los hogares con jefas femeninas, ya que permite identificar vulnerabilidades específicas de las que son objeto, y comparar dichas conformaciones con aquellas encabezadas por varones para identificar posibles desventajas que permitan encaminar 
de manera correcta esfuerzos en los análisis. Sobre los hogares con jefatura femenina, Echarri y López (2011) concluyen que

El incremento de la jefatura femenina es, sin duda, uno de los cambios más relevantes ocurridos en la organización de la vida familiar en los últimos años, no solo por su magnitud sino también por la fuerte carga cultural y simbólica que representa el hecho frente a las concepciones que se tienen sobre el arquetipo de familia nuclear compuesta por ambos padres y sus hijos. (Echarri y López, 2011, párr. 21)

El modelo de familia tradicional (hombre proveedor, mujer ama de casa), a partir del cual se han diseñado numerosas políticas, ha perdido vigencia; sin embargo, dado el contexto social muchas veces otras conformaciones familiares pueden estar en desventaja contra este "modelo de familia ideal". Para García y de Oliveira $(2011,2017)$ resulta necesaria la elaboración de estudios que profundicen en la relación de las transformaciones familiares sobre las relaciones de género y bienestar. Señalan que, en contextos desiguales, es imperativo realizar acercamientos que den cuenta de la heterogeneidad de las relaciones que se presentan en el interior de los hogares y que responden a los distintos arreglos familiares en los que estos se componen y a los estratos sociales a los que pertenecen ${ }^{9}$.

Respecto al sexo del jefe(a) del hogar y su impacto en el bienestar infantil, no existe un consenso claro sobre sus implicaciones. González de la Rocha (1999) expone que los hogares jefaturados por mujeres cuentan con un mayor número de perceptores de ingreso. Sin embargo, se identifica que las mujeres resultan las proveedoras principales de estas unidades y participan en mercados deteriorados donde históricamente se han visto relegadas, por lo que su bienestar y el de las unidades domésticas de las que se hacen cargo se pueden ver mermados.

También se ha documentado que en algunos hogares jefaturados por mujeres las estrategias de sobrevivencia implementadas consisten en el traspaso de la carga doméstica o de cuidados a los niños, niñas y adolescentes mayores que residan en el hogar (Acosta, 2001). Algunas veces su participación en el trabajo fuera del hogar es requerida y, dadas las reglamentaciones respecto al trabajo infantil vigentes alrededor del mundo, esto puede conllevar su introducción en empleos precarios y peligrosos.

\section{Metodología}

A continuación, se describe la fuente de información utilizada para realizar el análisis posterior; así mismo se profundiza en la descripción y construcción del modelo logístico

9 Si bien en este estudio no se profundiza en el estrato socioeconómico de pertenencia, la variable "promedio educativo del hogar" puede servir como proxi. 
multinomial y sobre la operacionalización de las variables empleadas en el análisis descriptivo y de los modelos desarrollados.

\section{Fuente de información}

La presente investigación es un análisis cuantitativo de carácter transversal, se emplea como fuente de información el Módulo de Trabajo Infantil (MTI) 2015 anexo a la ENOE de INEGI. El MTI 2015 cuenta con una muestra de 93493 niños, niñas y adolescentes, de estos 49429 tienen entre 5 y 11 años y 44064 entre 12 y 17 años. Dado que se excluyen del análisis a los NNA que habitan solos en los hogares; es decir, a los identificados como jefes del hogar, así como a los identificados como trabajadores domésticos o familiares de los trabajadores domésticos y a los huéspedes ${ }^{10}$, la muestra se redujo a 93 382. Por último, para la construcción de los modelos logísticos multinomiales se consideraron solo a aquellos NNA que cuentan con información válida para cada una de las variables independientes, por lo que la muestra de análisis es de 93157.

\section{Análisis estadístico}

Se efectuó un análisis estadístico exploratorio que al expandir las variables de la base la muestra de análisis representa un estimado de 29307910 NNA ${ }^{11}$. Con el fin de analizar de manera más profunda el perfil de los niños, niñas y adolescentes que trabajan en el ámbito doméstico y extradoméstico y estimar las probabilidades de realizar estos trabajos - respecto a no trabajar - se utilizaron tres modelos logísticos multinomiales (MLMN) multivariados, uno para la muestra analítica total de NNA, así como para aquellos que habitan en hogares con jefatura masculina y femenina. Según Agresti (2007), la regresión logística multinomial es una extensión de la regresión logística binomial, y esta se utiliza cuando la variable dependiente cuenta con más de dos categorías y en esta las variables explicativas (independientes) pueden ser categóricas o continuas.

Con base en lo expuesto por Long y Freese (2001), se presentan a continuación las características de estos modelos. Los autores exponen que a partir de los MLMN se pueden estimar de manera simultánea los logits binarios para todas las comparaciones entre las categorías dependientes:

$$
\ln \Omega \mathrm{m}\left|\mathrm{x}(\mathrm{x})=\ln \frac{\operatorname{Pr}(\mathrm{y}=\mathrm{m} \mid \mathrm{x})}{\operatorname{Pr}(\mathrm{y}=\mathrm{b} \mid \mathrm{x})}=\mathrm{x} \beta \mathrm{m}\right| \mathrm{b} \text { para } \mathrm{m}=1 \mathrm{a} \mathrm{J}
$$

Donde b es la categoría de referencia; es decir, el grupo de comparación y m representa las categorías de contraste. Dado que $\ln \Omega \mathrm{m} \mid x(x)=\ln 1=0$, se sostiene que $\beta b \mid b=0$. Esto

10 Se tomó esta decisión ya que un aspecto fundamental de este trabajo es dar cuenta del impacto de las características familiares en el hogar de residencia y su impacto sobre el trabajo en la infancia.

11 Este análisis puede verse en detalle en Valdez (2018). 
es, los odds de un resultado comparado con el mismo son siempre igual a 0 y, por lo tanto, los efectos de cualquier variable independiente deberían ser también 0 .

Siguiendo el ejemplo de Long y Freese (2001, p. 172), suponiendo que se tiene una variable dependiente con tres categorías $(\mathrm{M}, \mathrm{W}$ y $\mathrm{P})$ es posible estimar el efecto de las variables independientes sobre la dependiente al estimar tres logits binarios:

$$
\begin{aligned}
& \ln \frac{\operatorname{Pr}(y=P \mid x)}{\operatorname{Pr}(y=M \mid x)}=\beta_{0, P \mid M}+\beta_{1, P \mid M} V A R \\
& \ln \frac{\operatorname{Pr}(y=W \mid x)}{\operatorname{Pr}(y=M \mid x)}=\beta_{0, W \mid M}+\beta_{1, W \mid M} V A R \\
& \ln \frac{\operatorname{Pr}(y=P \mid x)}{\operatorname{Pr}(y=W \mid x)}=\beta_{0, P \mid W}+\beta_{1, P \mid W} V A R
\end{aligned}
$$

Es posible demostrar que los modelos expuestos son redundantes y que, por lo tanto, se pueden ajustar a J-1, pues:

$$
\ln \frac{\operatorname{Pr}(y=P \mid x)}{\operatorname{Pr}(y=W \mid x)}=\beta_{0, P \mid W}+\beta_{1, P \mid W} V A R
$$

$\ln \frac{a}{b}=\ln a-\ln b ;$ es decir:

$$
\ln \frac{\operatorname{Pr}(y=P \mid x)}{\operatorname{Pr}(y=W \mid x)}-\ln \frac{\operatorname{Pr}(y=W \mid x)}{\operatorname{Pr}(y=M \mid x)}=\ln \frac{\operatorname{Pr}(y=P \mid x)}{\operatorname{Pr}(y=W \mid x)}
$$

Lo que implica que:

$$
\begin{aligned}
& \beta_{0, P \mid M}-\beta_{0, W \mid M}=\beta_{0, P \mid W} \\
& \beta_{1, P \mid M}-\beta_{1, W \mid M}=\beta_{1, P \mid W}
\end{aligned}
$$

Resulta entonces innecesario estimar los tres modelos, por lo que estos se ajustan a J-1 modelos logísticos binarios.

Se pueden estimar las probabilidades de que algún individuo pertenezca a alguna de las categorías de la variable dependiente lo cual se puede expresar mediante la siguiente ecuación:

$$
\operatorname{Pr}(y=m \mid x)=\frac{e^{x \beta m \mid b}}{\Sigma j=1 J e^{x \beta j \mid b}}
$$

En estas ecuaciones los subíndices de las $\beta$ indican las comparaciones que se realizan. 
Fueron identificadas algunas limitaciones en la fuente de datos, además de las limitaciones naturales de una encuesta representativa (nivel geográfico de análisis, posibles errores de muestreo, especificidad del tema, etc.) (Tabutin, 1997). Debe mencionarse que la temática del trabajo infantil genera por sí misma situaciones particulares en los levantamientos, pues al ser una actividad prohibida y con fuertes cargas sociales se puede evitar la recolección de información o comprometer la calidad de los datos (Miranda y Navarrete, 2016). Por último, es necesario considerar que normalmente es un tercero quien reporta las actividades que realizan niños, niñas y adolescentes, debido a las limitaciones establecidas en la edad para responder una encuesta, las respuestas corresponden a las percepciones de otros miembros del hogar y no necesariamente a las vivencias propias del grupo de estudio.

\section{Variables}

Para categorizar a los niños niñas y adolescentes según su condición laboral, es decir, para la construcción de la variable dependiente, se utilizan las variables precodificadas en el MTI 2015. Los NNA que realizan trabajo en la infancia en el ámbito extradoméstico comprenden a los que se clasifican bajo la edad mínima de acceso al empleo y a los que se encuentran en las llamadas ocupaciones peligrosas ${ }^{12}$. En cuanto al trabajo en la infancia en el ámbito doméstico este se construye con NNA que realizan quehaceres domésticos en horarios prolongados y en condiciones peligrosas ${ }^{13}$. Por último, aquellos NNA que no realizan trabajo en la infancia doméstico o extradoméstico se categorizan como "no realiza trabajo en la infancia".

Se definen tres bloques de variables para el estudio: macro (contextual), meso (familiar) y micro (individual). A continuación, se explican los criterios de agrupación de las variables de control y de interés:

A nivel macro (contextual):

- Tamaño de localidad. Cuenta con cuatro categorías que hacen referencia al número de habitantes: más de 100000 , entre $15000-99999$, entre 2500-14999 y menos de 2500 .

12 Quienes realizan trabajo bajo la edad mínima son los niños y niñas de entre 5 y 14 años, según el artículo 123 de la Constitución Federal de los Estados Unidos Mexicanos. La definición de los NNA en ocupaciones peligrosas es más amplia, pues contempla que realicen actividades en lugares peligrosos e insalubres, que las actividades reportadas los expongan a riesgos sobre su salud o moral, que no cuenten con permisos y certificados de salud y que realicen actividades en horarios nocturnos o prolongados.

13 En el caso de los horarios prolongados se consideran los siguientes criterios para su clasificación: a) 5 a 11 años, superior a 14 horas; b) de 12 a 14 años, mayor de 28 horas; c) 15 años, más de 36 horas; y d) 16 y 17 años mayor a 48 horas. Para las condiciones peligrosas se integran los NNA para los que se declara que por la actividad que realizan deben cargar cosas pesadas, sufren de dolores, cansancio o agotamiento, presentan problemas respiratorios o no cumple con sus actividades escolares. 
A nivel meso (características del hogar y del jefe(a) de esta):

- Clase de hogar. Cuenta con tres categorías: jefe(a) e hijos; pareja e hijos, y otros tipos de hogar (extensos, compuestos y no familiares).

- $\quad$ Presencia de niños menores de 5 años en el hogar. Variable dicotómica que indica si habitan o no habitan personas de esta edad.

- Presencia de adultos mayores de 65 años en el hogar. Variable dicotómica que indica si habitan o no habitan personas de esta edad.

- $\quad$ Sexo del jefe(a) del hogar. Indica si es hombre o mujer.

- Condición de ocupación del jefe(a) del hogar: ocupaciones no manuales, manuales (exceptuando agrícolas), agrícolas y jefe(as) no ocupados.

- Promedio educativo del hogar: promedio de los años educativos alcanzados por las personas de 18 años y más que residen en el hogar ${ }^{14}$.

A nivel micro (características individuales de NNA):

- Condición de asistencia escolar. Variable dicotómica que indica si los NNA asisten o no asisten a la escuela.

- Posición en el hogar. Conjunta el sexo y la edad de los NNA en el hogar e identifica a los niños y niñas mayores, niños posteriores y niñas posteriores.

\section{Resultados}

En este apartado se presentan los resultados tanto descriptivos como del modelo logístico multinomial, para la muestra de estudio total de niños, niñas y adolescentes; así como para dos submuestras, aquellos que habitan en hogares con jefatura masculina y femenina, de acuerdo con la condición de trabajo dentro y fuera de los hogares - o de no trabajo - del grupo de estudio.

A nivel macrocontextual, se identificó que al reducirse el tamaño de la localidad se incrementó la proporción de NNA que realizaron actividades domésticas y extradomésticas en la muestra total y en las submuestras analizadas; cabe mencionar que la participación en valores relativos y para los tres grupos incrementó en mayor medida en el trabajo extradoméstico (tabla 1).

A nivel meso (familiar), se observó que para el tipo de hogar las unidades conformadas por pareja e hijos presentan una proporción menor de NNA que realizan trabajo en los 
ámbitos doméstico y extradoméstico que quienes habitan en unidades monoparentales. Por otro lado, cohabitar con adultos mayores en el hogar no se tradujo en diferencias porcentuales importantes en lo referente al trabajo fuera de los hogares; sin embargo, en los tres grupos de análisis, la proporción de NNA que realizaron trabajo doméstico se vio reducida en poco más de un punto porcentual. Contrario a este resultado, las proporciones de participación en trabajo doméstico se incrementó con la presencia de niños menores de cinco años en el hogar.

El promedio educativo de los hogares fue superior cuando se declaró que los NNA no realizaron actividades domésticas o extradomésticas. En el caso de las actividades extradomésticas, dicho promedio se redujo en casi dos años respecto a quienes no realizaron la actividad. En lo referente a la ocupación del jefe(a) del hogar, para ambos tipos de trabajo, el desempeñar actividades agrícolas incrementó de manera importante la proporción de NNA que realizaron trabajo extradoméstico. Por último, existe una mayor proporción de NNA que realizaron actividades domésticas y extradomésticas y habitaban en hogares con jefatura femenina.

A nivel individual, el no asistir a la escuela mostró las proporciones más elevadas de NNA que realizaron ambos tipos de trabajo. Finalmente, ser la niña mayor del hogar expuso las proporciones más elevadas de realización de trabajo doméstico, mientras que ser el primer niño reflejó las más elevadas para el trabajo extradoméstico, tendencias similares para ambas submuestras. 


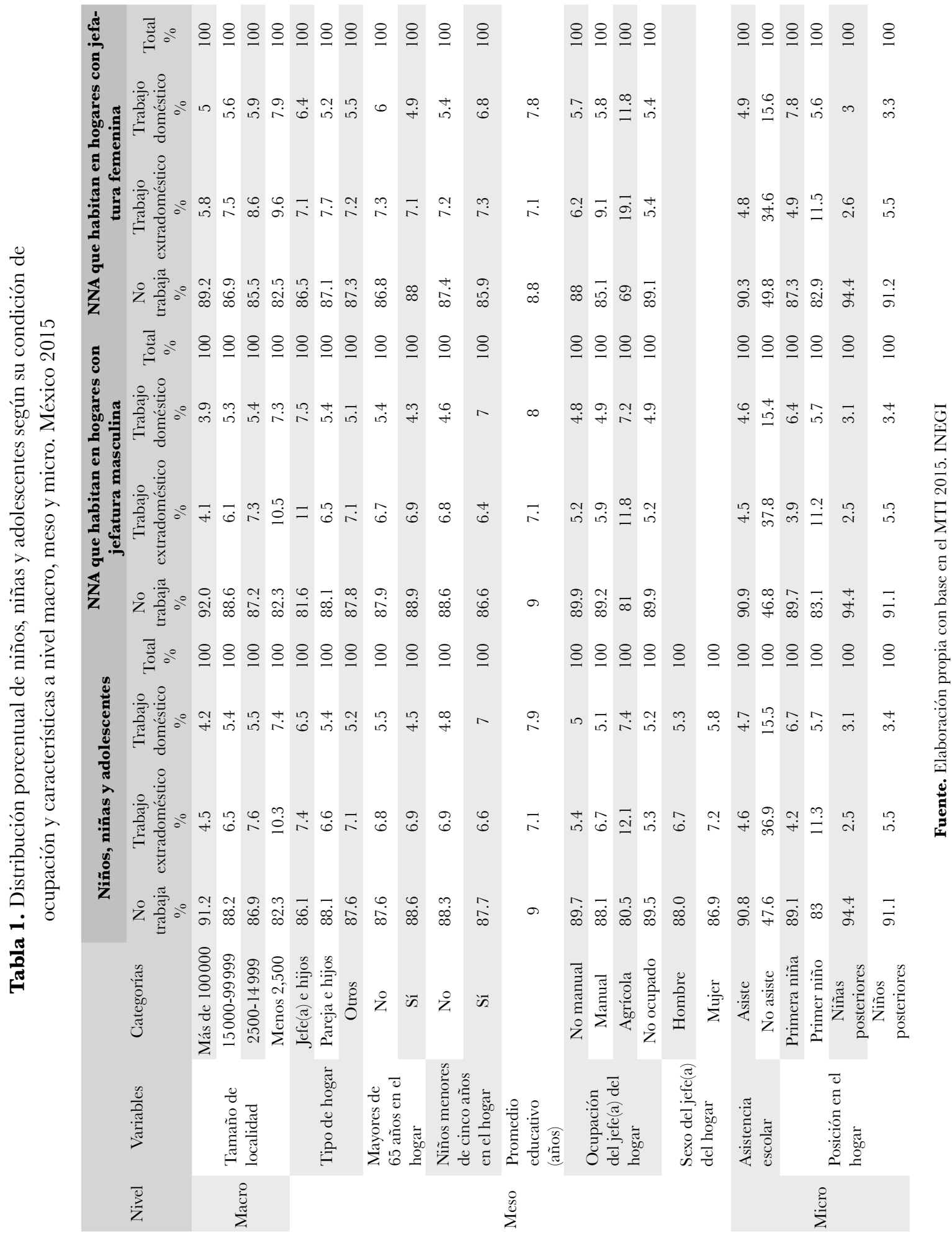


En la tabla 2 se muestran los resultados de los modelos logísticos multinomiales, donde es posible identificar los factores determinantes del trabajo en la infancia; es decir, se evalúa el poder explicativo de las variables sobre la población infantil y adolescente en las tres categorías de análisis: 1) no realiza trabajo en la infancia, 2) realiza trabajo extradoméstico y 3) realiza trabajo doméstico.

En el modelo general, a nivel macro se encontró que al reducirse el tamaño de la localidad incrementó la probabilidad de realizar ambos tipos de trabajo. Las áreas más urbanizadas — las de más de 100.000 habitantes — exhibieron las probabilidades más bajas $(5.6 \%)$ de realizar trabajo extradoméstico, y aquellas con menos de 2500 habitantes, las mayores $(7.9 \%)$. De igual manera, para el trabajo realizado dentro de los hogares fueron las localidades más pequeñas — de menos de 2500 habitantes - las que mostraron una mayor probabilidad $(6.5 \%$ ) y las más urbanizadas — de más de 100000 - la probabilidad más reducida $(4.7 \%)$.

En lo referente al arreglo del hogar, esta composición no se identificó como un factor determinante, pues las probabilidades de realizar trabajo extradoméstico no mostraron diferencias significativas entre uno y otro tipo de confirmación $(6.9 \%$ para los NNA que habitaban en hogares con pareja e hijos, $6.8 \%$ en hogares monoparentales y 6.7 $\%$ en otro tipo de arreglos). Sin embargo, estas tendencias resultaron distintas en el trabajo doméstico, pues infantes y adolescentes que habitaban en hogares monoparentales tuvieron la probabilidad más alta de efectuar este tipo de trabajo (6.6\%), seguidos por aquellos que residían en unidades compuestas por parejas e hijos (5.5\%), por último, los que vivían en otros tipos de arreglos del hogar $(4.9 \%)$.

El habitar con adultos mayores de 65 años en los hogares no fue un factor determinante para explicar que NNA se involucraran en alguno de los dos tipos de trabajo. No obstante, el que no habitaran menores de 5 años en la unidad doméstica presentó una probabilidad más alta de participación en el trabajo extradoméstico, que aquellos que corresidían con este grupo poblacional ( $7.1 \%$ y $6.2 \%$ respectivamente). Por otro lado, esta probabilidad tuvo una relación inversa en lo referente al trabajo doméstico, pues la corresidencia de niños menores de 5 años mostró una mayor probabilidad de realizar trabajo doméstico $(6.7 \%)$, la cual se redujo en su ausencia $(4.9 \%)$.

En lo referente a las características del jefe(a) de hogar, quienes habitan en unidades lideradas por mujeres presentaron una probabilidad más elevada de realizar trabajo extradoméstico $(7.9 \%$ ), comparados con quienes residían en unidades jefaturadas por varones $(6.5 \%)$. No obstante, la probabilidad de realizar trabajo doméstico no presentó diferencias significativas entre ambas unidades; solo se identificó una diferencia de 0.5 puntos porcentuales entre los hogares encabezados por mujeres $(5.8 \%$ ) y los dirigidos por varones $(5.3 \%)$.

Cuando el jefe(a) del hogar se involucraba en actividades agrícolas, NNA presentaron una probabilidad superior $(8.5 \%)$ de integrarse en el trabajo extradoméstico, seguidos 
por aquellos cuyo jefe(a) declaró realizar actividades manuales $(7.4 \%)$ y no manuales $(6.5 \%$ ). Finalmente, el que los jefes(as) del hogar no se encontraran ocupados mostró la probabilidad más baja (4.6\%). Sin embargo, en el caso del trabajo doméstico no se encontraron diferencias importantes entre estas categorías, por lo que el tipo y condición de ocupación parece no ser determinante.

Respecto a los factores individuales, la inasistencia escolar mostró la probabilidad más elevada de realizar trabajo extradoméstico (30.5\%) y doméstico (14\%), así como ser el niño de mayor edad que habita en el hogar (10.9\%). Contrario a lo que se encuentra en el trabajo extradoméstico, se observa que son las niñas y los niños mayores en el hogar los que presentaron las probabilidades más altas (6.6\% y $5.7 \%$ respectivamente).

\section{Niños en hogares con jefatura masculina}

En lo referente a los modelos realizados para las submuestras, los resultados reflejaron para los niños que habitan en hogares con jefatura masculina que en las localidades más urbanizadas - 100000 y más habitantes- la probabilidad de realizar trabajo extradoméstico y doméstico fue de $5.2 \%$ y $4.4 \%$, respectivamente; por su parte, en las áreas de menor tamaño - 2500 habitantes- se localizan las probabilidades superiores $8.0 \%$ y $6.4 \%$.

Respecto a la configuración de los hogares, las unidades monoparentales presentaron la mayor probabilidad de realizar ambos tipos de trabajo $(8.4 \%$ para el trabajo extradoméstico y $7.8 \%$ para el doméstico); por el contrario, las probabilidades de realizar trabajo extradoméstico fueron diferentes al habitar en hogares donde residían parejas e hijos u en otros tipos de arreglo familiar. Sin embargo, otros tipos de arreglo beneficiaron en mayor medida a NNA en lo que respecta al trabajo realizado dentro de la esfera doméstica $(4.7 \%)$.

$\mathrm{Al}$ igual que en el modelo general, la residencia con adultos mayores de 65 años redujo la probabilidad de trabajar en el ámbito doméstico $(5.4 \%$ para los niños que habitan en hogares donde no residían adultos mayores y 4.6 \% para los que sí comparten residencia), y no existieron diferencias importantes en lo referente a dicho trabajo doméstico. Respecto a la presencia de menores de 5 años en el hogar, la probabilidad de realizar trabajo extradoméstico para los NNA que no habitaban con menores de esta edad fue de $7.1 \%$, y para los que sí lo hacen de $6.0 \%$; esta variable opera en sentido contrario respecto al trabajo doméstico, ya que se aumentaron las probabilidades para los que sí comparten residencia $(6.7 \%$ y se reducen para los que no lo hacen $(4.7 \%)$.

Referente a la ocupación del jefe de hogar, la probabilidad de realizar trabajo extradoméstico es mayor para los niños que habitan en hogares donde este se desarrolla en el ámbito agrícola $(7.9 \%$ ), seguida por los que realizan actividades manuales y no manuales (6.8 \% y $6.2 \%$ ), finalmente, para los que no se encuentran ocupados $(5.0 \%)$. En cuanto a las probabilidades de realizar trabajo doméstico, para esta muestra las 
categorías de ocupación del jefe no mostraron diferencias estadísticas importantes que permitan inferirlas como un factor explicativo del fenómeno.

La condición de asistencia escolar exhibió la probabilidad más alta en el modelo para ambos tipos de trabajo: $30.7 \%$ en el caso del extradoméstico y $14.0 \%$ en el del doméstico. Respecto a la posición en el hogar, ser el varón de mayor edad sostuvo la probabilidad más alta de realizar trabajo extradoméstico (10.8\%), seguido de los varones posteriores $(6.1 \%$ ) y de las primeras niñas $(3.9 \%)$; sin embargo, las probabilidades del trabajo doméstico fueron superiores para las primeras niñas $(6.3 \%)$, seguidas de los primeros varones $(5.7 \%)$. Las brechas más altas en esta categoría se presentaron entre las niñas y niños mayores de edad en el trabajo extradoméstico. La probabilidad de no realizar ninguno de los trabajos fue superior para las niñas más pequeñas del hogar (3.0 $\%$ para el trabajo extradoméstico y $3.3 \%$ para el doméstico).

\section{Niños en hogares con jefatura femenina}

Para el grupo de estudio residente en hogares con jefatura femenina, las probabilidades de realizar trabajo extradoméstico fueron inferiores en las localidades de más de 100000 habitantes (6.5 \%), y superiores en las de entre 2500 y 14999 (8.5\%). El trabajo doméstico siguió la dirección esperada donde se incrementan las probabilidades al reducir el tamaño de la localidad; esta fue de $5.4 \%$ para las áreas más urbanizadas y $6.8 \%$ para las de menor tamaño.

En lo concerniente a la organización familiar, quienes habitaban en hogares encabezados por mujeres con pareja tienen una probabilidad mayor de realizar trabajo extradoméstico $(8.5 \%)$ que en los monoparentales $(7.3 \%$ y en otros tipos de arreglos $(6.9 \%)$. Este resultado difiere al de NNA que residían en unidades con jefatura masculina. En cuanto al trabajo doméstico, las unidades monoparentales exhibieron la mayor probabilidad de participación infantil $(6.6 \%)$, mientras que la más baja se encontró en otros tipos de arreglo del hogar $(5.3 \%)$.

El hecho de que en la unidad doméstica habiten adultos mayores de 65 años mostró una mayor probabilidad de emplearse en el trabajo extradoméstico (8.0\%) respecto a los hogares donde no residían (7.1\%), y se redujo al realizar trabajo doméstico $(5.4 \%$ en donde sí residen y $5.9 \%$ en donde no). Vivir con niños menores de 5 parece no tener efectos importantes en cuanto al trabajo extradoméstico, pero su presencia mostró una probabilidad superior en cuanto a las labores realizadas dentro del hogar $(6.6 \%$ versus $5.5 \%$ donde no hay niños menores).

La variable ocupación del jefe del hogar presentó el mismo comportamiento para los niños que residían en hogares con jefatura masculina. El trabajo agrícola expuso las mayores probabilidades de realizar trabajo doméstico y extradoméstico $(11.8 \%$ y $8.8 \%$, 
respectivamente); sin embargo, en este tipo de trabajo existieron brechas más pronunciadas en el caso de las unidades jefaturadas por mujeres.

La inasistencia escolar, a nivel individual, fue el factor que predominó en mayor medida en ambos tipos de trabajo con probabilidades de $29.8 \%$ y $13.9 \%$ para el trabajo extradoméstico y doméstico, respectivamente. La manera en que se posicionan NNA en los hogares presentó efectos en el mismo sentido que en los hogares con jefatura masculina para ambos tipos de trabajo; en el caso del extradoméstico, las probabilidades más elevadas estuvieron en los primeros varones (11.2\%), seguidos de los niños (varones) posteriores (6.4\%) y de las mujeres mayores en el hogar ( $4.7 \%)$; no obstante, en el trabajo doméstico son las niñas mayores quienes mostraron una mayor probabilidad $(7.7 \%)$. 
Tabla 2. Niños, niñas y adolescentes de 5 a 17 años. Probabilidades asociadas (\%) del modelo de regresión logística multinomial de realizar trabajo doméstico, extradoméstico o no realizar trabajo en la infancia por sexo del jefe(a) del hogar*

\begin{tabular}{|c|c|c|c|c|c|c|c|c|c|c|}
\hline \multirow[b]{2}{*}{ Variable } & \multirow[b]{2}{*}{ Categorías } & \multicolumn{3}{|c|}{ General } & \multicolumn{3}{|c|}{ Jefatura masculina } & \multicolumn{3}{|c|}{ Jefatura femenina } \\
\hline & & TED & $\mathrm{TD}$ & $\begin{array}{l}\text { No } \\
\text { realiza } \\
\text { TI }\end{array}$ & TED & $\mathrm{TD}$ & $\begin{array}{l}\text { No } \\
\text { realiza } \\
\text { TI }\end{array}$ & TED & TD & $\begin{array}{l}\text { No } \\
\text { realiza } \\
\text { TI }\end{array}$ \\
\hline \multirow{4}{*}{$\begin{array}{l}\text { Tamaño de la } \\
\text { localidad }\end{array}$} & Más de 100000 & 5.6 & 4.7 & 89.8 & 5.2 & 4.4 & 90.4 & 6.5 & 5.4 & 88.1 \\
\hline & $15000-99999$ & 7 & 5.5 & 87.5 & 6.7 & 5.5 & 87.7 & 7.6 & 5.6 & 86.7 \\
\hline & 2500-14999 & 7.5 & 5.4 & 87.1 & 7.2 & 5.3 & 87.5 & 8.5 & 5.9 & 85.7 \\
\hline & Menos de 2500 & 7.9 & 6.5 & 85.6 & 8 & 6.4 & 85.6 & 7.7 & 6.8 & 85.5 \\
\hline \multirow{3}{*}{$\begin{array}{l}\text { Composición del } \\
\text { hogar }\end{array}$} & Pareja e hijos & 6.9 & 5.5 & 87.6 & 6.7 & 5.5 & 87.8 & 8.5 & 5.5 & 86.1 \\
\hline & Jefe(a) e hijos & 6.8 & 6.6 & 86.5 & 8.4 & 7.8 & 83.7 & 7.3 & 6.6 & 86.1 \\
\hline & Otros arreglos & 6.7 & 4.9 & 88.4 & 6.7 & 4.7 & 88.5 & 6.9 & 5.3 & 87.9 \\
\hline \multirow{2}{*}{$\begin{array}{l}\text { Mayores de } 65 \\
\text { años en el hogar }\end{array}$} & No & 6.8 & 5.5 & 87.7 & 6.8 & 5.4 & 87.8 & 7.1 & 5.9 & 87 \\
\hline & Sí & 6.7 & 4.9 & 88.4 & 6.2 & 4.6 & 89.2 & 8.0 & 5.4 & 86.6 \\
\hline \multirow{2}{*}{$\begin{array}{l}\text { Menores de } 5 \\
\text { años en el hogar }\end{array}$} & No & 7.1 & 4.9 & 88 & 7.1 & 4.7 & 88.2 & 7.3 & 5.5 & 87.2 \\
\hline & Sí & 6.2 & 6.7 & 87.2 & 6 & 6.7 & 87.4 & 7.1 & 6.6 & 86.3 \\
\hline \multirow{2}{*}{$\begin{array}{l}\text { Sexo del jefe(a) } \\
\text { del hogar }\end{array}$} & Hombre & 6.5 & 5.3 & 88.1 & & & & & & \\
\hline & Mujer & 7.9 & 5.8 & 86.3 & & & & & & \\
\hline \multirow{4}{*}{$\begin{array}{l}\text { Ocupación del } \\
\text { jefe de hogar }\end{array}$} & No manual & 6.5 & 5.5 & 88 & 6.2 & 5.3 & 88.5 & 7.5 & 6.2 & 86.3 \\
\hline & Manual & 7.4 & 5.4 & 87.2 & 6.8 & 5.3 & 87.9 & 9.4 & 5.8 & 84.8 \\
\hline & Agrícola & 8.5 & 5.7 & 85.7 & 7.9 & 5.5 & 86.6 & 11.8 & 8.8 & 79.4 \\
\hline & No ocupado & 4.6 & 5.1 & 90.3 & 5.0 & 5.3 & 89.7 & 5.0 & 5.3 & 89.7 \\
\hline \multirow{2}{*}{$\begin{array}{l}\text { Condición de } \\
\text { asistencia escolar }\end{array}$} & Asiste & 4.7 & 4.8 & 90.5 & 4.6 & 4.7 & 90.7 & 4.9 & 5.0 & 90.0 \\
\hline & No asiste & 30.5 & 14 & 55.5 & 30.7 & 14 & 55.3 & 29.8 & 13.9 & 56.3 \\
\hline \multirow{4}{*}{$\begin{array}{l}\text { Posición en el } \\
\text { hogar }\end{array}$} & Primera niña & 4.1 & 6.6 & 89.3 & 3.9 & 6.3 & 89.8 & 4.7 & 7.7 & 87.6 \\
\hline & Primer niño & 10.9 & 5.7 & 83.5 & 10.8 & 5.7 & 83.5 & 11.2 & 5.6 & 83.3 \\
\hline & Niñas posteriores & 3.0 & 3.3 & 93.7 & 3.0 & 3.3 & 93.7 & 3.1 & 3.2 & 93.7 \\
\hline & Niños posteriores & 6.2 & 3.5 & 90.3 & 6.1 & 3.5 & 90.4 & 6.4 & 3.5 & 90.2 \\
\hline
\end{tabular}

* Nota: la probabilidad estimada se calculó como la probabilidad esperada de un NNA con características promedio en cada una de las variables.

TED: trabajo extradoméstico, TD: trabajo doméstico, TI: trabajo infantil.

Fuente. Cálculos propios con base en el MTI (2015) 


\section{Discusión y conclusiones}

La conceptualización y, por lo tanto, la operacionalización de variables referentes al trabajo infantil es un ejercicio complicado, pues hasta el año 2008 se establecieron a nivel institucional acuerdos para su delimitación conceptual y su medición estadística, particularmente en lo referente al trabajo extradoméstico, pues la discusión de su definición continúa vigente. Es necesario profundizar en la manera en que se conceptualiza el trabajo doméstico que realizan NNA y su valor social, así como en implementar instrumentos con mayor solidez que permiten captar de manera directa las respuestas de NNA sobre las actividades que llevan a cabo y sus percepciones respecto a estas.

En esta investigación el trabajo en la infancia se definió bajo los criterios de medición que permitieron la clasificación y construcción de variables específicas en el MTI 2015. Bajo estos marcos operativos fue posible construir los modelos estadísticos y determinar en qué medida los factores de análisis seleccionados inciden en que niños, niñas y adolescentes realicen trabajo en la infancia.

El objetivo principal de la investigación fue determinar de qué manera el tipo de arreglo familiar, como una variable a nivel meso, se relacionaba con la integración de NNA al trabajo extradoméstico y doméstico e identificar si dicha integración se diferencia de acuerdo con el sexo del jefe(a); al respecto, los resultados indican que existen riesgos diferenciados de realizar trabajo en la infancia en el modelo general, al analizar los riesgos relativos de hacer trabajo extradoméstico, no se encontraron diferencias significativas entre los hogares monoparentales y aquellos donde residían parejas e hijos. Así mismo, otros tipos de arreglo familiar (extensos, compuestos y no familiares) tampoco mostraron efectos que permitan establecer diferencias con otras conformaciones. Resultados similares son reportados por Miranda y Navarrete (2016) para el grupo de niños y niñas de entre 5 y 11 años. Estos hallazgos sugieren que la vulneración de los ingresos familiares prevalece como detonante de la integración al mercado laboral en menores de 18 años, esto por encima de la manera en que se constituyen los hogares.

Contrario a lo anterior, la probabilidad de realizar trabajo doméstico fue diferente en cada una de las conformaciones, mientras que la residencia de NNA en hogares monoparentales muestra una mayor probabilidad de realizarlo, mientras que en otro tipo de arreglos esta es más reducida. Al respecto, se ha identificado que la participación de NNA en tareas del hogar puede compensar la falta de un miembro no residente (padre o madre) (Demo y Acock, 1993), ya que mientras el padre o la madre fungen como proveedores principales, NNA podrían suplirlos en la realización de las actividades domésticas como parte de las estrategias de vida de los hogares. Dentro de este mecanismo de sustitución quienes tienen un mayor riesgo de tener una participación alta en el trabajo en el hogar son las niñas y las adolescentes frente a sus pares del sexo masculino. Esto último evidencia la reproducción 
de roles de género al momento de integrarse a las labores cotidianas realizadas en la esfera doméstica.

Por otro lado, los hogares extensos y compuestos (integrados en otros arreglos) cuentan normalmente con una mayor cantidad de miembros, por lo que se podría suponer que existe una distribución importante de las tareas dentro de las unidades, que impide una sobrecarga de trabajo doméstico o que la realización de labores peligrosas recaiga sobre los NNA.

Los resultados del modelo general muestran que niños, niñas y adolescentes que habitan en hogares con jefatura femenina presentan propensiones más elevadas a realizar ambos tipos de trabajo que sus contrapartes en hogares con jefatura masculina. Estas probabilidades evidencian una brecha más elevada en lo referente al trabajo extradoméstico. El sistema económico, social y político ha propiciado que se establezcan roles de acuerdo con el género de cada individuo, dichos roles han relegado, tradicionalmente, a las mujeres al especio privado (hogar), mientras que han concedido una mayor participación pública a los hombres. Sin embargo, en un proceso de continuas transformaciones sociales, las mujeres han incrementado su participación en la esfera pública, pero no necesariamente la han reducido en la privada. Si bien en este momento no es tema de discusión la manera en que hombres y mujeres perciben y sobrellevan dichas imposiciones, sería importante analizar en el futuro la manera en que niños, niñas y adolescentes las internalizan.

El trabajo extradoméstico se encuentra de alguna manera regulado, al menos en términos legales, el hecho de que el trabajo doméstico se realice dentro de la esfera privada plantea retos importantes, no solo para su medición, sino para la aplicación de regulaciones que permitan proteger a NNA de labores que pueden vulnerarlos. El que los hogares con jefatura femenina presenten los riesgos más elevados para su realización es un llamado de atención a poner como foco de interés las vulnerabilidades específicas que atraviesa este tipo de conformaciones.

\section{Referencias bibliográficas}

Acosta, F. (2001). Jefatura de hogar femenina y bienestar familiar: resultados de la investigación empírica. Papeles de población, 7(28), 41-97. Recuperado de http://repositoriodigital.academica.mx/jspui/handle/987654321/218284

Agresti, A. (2007). Multicategory Logit Models. An Introduction to Categorical Data Analysis. New Jersey: JohnWiley \& Sons, Inc. DOI: https://doi.org/10.1002/0471249688

Ariza, M. y Oliveira, O. (2001). Familias en transición y marcos conceptuales en redefinición. Papeles de población, 7(28), 9-39. Recuperado de http://www.scielo.org.mx/ scielo.php?script $=$ sci_arttext\&pid=S1405-74252001000200002 
Ariza, M. y Oliveira, O. (2007). Familias, pobreza y desigualdad social en Latinoamérica : una mirada comparativa. Estudios Demográficos y Urbanos, 22(1), 9-42.

Briceño, L. y Pinzón, Á. M. (2004). Efectos del trabajo infantil en la salud del menor trabajador. Revista de Salud Pública, 6, 270-288.

Contreras, F. (2017). Dinámica laboral en los hogares rurales de México. En J. Nájera, B. García, y E. Pacheco (eds.), Hogares y trabajadores en México del siglo XXI (pp. 189226). Ciudad de México: El Colegio de México.

Demo, D. H. y Acock, A. C. (1993). Family diversity and the division of domestic labor: How much have things really changed? Family Relations, 323-331.

Diario Oficial de la Federación [DOF]. (2014). Constitución Política de los Estados Unidos Mexicanos. Título 6. del trabajo y la previsión social. Artículo 123. Distrito Federal.

Echarri, C. J. y López, M. (2011). Hogares, vivienda y jefatura femenina. Recuperado de http:/ / archivo.estepais.com/site/2011/hogares-vivienda-y-jefatura-femenina/

Edgell, S. (2011). The Historical Transformation of Work. The sociology of work: Continuity and change in paid and unpaid work. SAGE.

Estrada, L. (2005). Familia y trabajo infantil y adolescente en México, 2000. En M. Mier y C. Rabell (eds.), fóvenes y niños: un enfoque sociodemográfico (pp. 203-247). México D. F.: UNAM, FLACSO: Porrúa.

García, B. (2019). El trabajo doméstico y de cuidado: su importancia y principales hallazgos en el caso mexicano. Estudios Demográficos y Urbanos, 34(2), 237-267.

García, B. y de Oliveira, O. (2011). Cambios familiares y políticas públicas en América Latina. Annual Review of Sociology, 37(1), 613-633. DOI: https://doi.org/10.1146/ annurev-soc-033111-130034

García, B. y de Oliveira, O. (2014). Familias, trabajo y políticas: encuentros y desencuentros. En S. Giorguli y V. Ugalde (coords.), Gobierno, territorio y población: las políticas públicas en la mira (pp. 195-232). México: El Colegio de México

González de la Rocha, M. (1999). Hogares de jefatura femenina en México: patrones y formas de vida. En M. González de la Rocha (ed.), Divergencias del modelo tradicional: hogares de jefatura femenina en América Latina (pp. 1-12). Plaza y Valdés: Guadalajara.

Instituto Nacional de Estadística y Geografía [INEGI]. (2015). Encuesta intercensal. Recuperado de https://www.inegi.org.mx/programas/intercensal/2015/ 
Revista CIFE / ISSN: 0124-3551 e-ISSN: 2248-4914 / Bogotá-Colombia / Vol. 21 N.o 34 / enero-junio 2019 / pp. 47-68

Instituto Nacional de Estadística y Geografía [INEGI]. (2016). Módulo de Trabajo Infantil (MTI) 2015 Encuesta Nacional de Ocupación y Empleo. Recuperado de https:/ / www.inegi.org.mx/programas/mti/2015/default.html

Long, S. y Freese, J. (2001). Models for Nominal Outcomes. Categorical Dependent Variables Using Stata). Texas: UCLA.

Mier, M., Terán, M. y Rabell, C. (2004). Familia y quehaceres entre los jóvenes. En M. Mier, M. Terán y O. De Oliveira (eds.), Imágenes de la familia en el cambio de siglo (pp. 135-179). Ciudad de México: Instituto de Investigaciones Sociales-Universidad Nacional Autónoma de México.

Miranda, S. y Navarrete, E. L. (2016). El entorno familiar y el trabajo de niñas y niños de 5 a 11 años. México en dos momentos: 2007 y 2013. Papeles de población, 22(89), 43-72. Recuperado de http://www.scielo.org.mx/scielo. php?pid=S1405-74252016000300043\&script=sci_arttext

Oliveira, O. y García, B. (2017). Aproximaciones sociodemográficas al estudio de los hogares y familias en México. En J. Nájera, B. García y E. Pacheco (eds.), Hogares y trabajadores en México del siglo XXI (pp. 71-128). México: El Colegio de México.

Organización Internacional del Trabajo [OIT]. (2006). La eliminación del trabajo infantil: un objetivo a nuestro alcance. Informe $I(B)$. Ginebra: Organización Internacional del Trabajo. Recuperado de http://www.ilo.org/public/spanish/standards/relm/ilc/ ilc95/pdf/rep-i-b.pdf

Organización Internacional del Trabajo [OIT]. (2008). Segunda resolución del informe de la Decimoctava Conferencia Internacional de Estadísticos del Trabajo. Ginebra, Suiza. Recuperado de http://www.ilo.org/wcmsp5/groups/public/---dgreports/---stat/ documents/meetingdocument/wcms_101551.pdf

Pérez, M. J. (2015). El trabajo infantil, institucionalización de su regulación y avances para su medición. En A. Hernández y A. E. Campos (eds.), Actores, redes y desafios: Juventudes e infancias en América Latina (pp. 247-266). Tijuana, Baja California: Consejo Latinoamericano de Ciencias Sociales.

Tabutin, D. (1997). Sistemas de información en demografía. Estudios Demográficos y Urbanos, 12(3), 377-426.

Valdez, C. (2018). Trabajo en la infancia en los ámbitos doméstico y extradoméstico: una aproximación desde las características de los hogares. México: El Colegio de México. 